\title{
Low post-pandemic wave SARS-CoV-2 seroprevalence in Kuala Lumpur and Selangor, Malaysia
}

\author{
I-Ching Sam ${ }^{1}$, Yoong Min Chong ${ }^{1}$, Chee Wah Tan ${ }^{2}$, and Yoke Fun Chan ${ }^{1}$ \\ ${ }^{1}$ University Malaya \\ ${ }^{2}$ Duke-NUS Medical School Program in Emerging Infectious Diseases
}

August 12, 2020

To the Editor,

Seroprevalence studies provide a more accurate picture of coronavirus disease 2019 (COVID-19) than PCRconfirmed cases as antibodies can be detected in mild or asymptomatic cases who otherwise remain undiagnosed. Seroprevalence can also be used as an indicator of population immunity. The majority of seroprevalence studies to date have been carried out in developed countries. High levels of herd immunity were recently estimated to be needed to control the spread of COVID-19 in different countries, including Malaysia. ${ }^{1}$

The first COVID-19 case in Malaysia was reported on January 25, 2020, and the main wave occurred between early March and mid-April. With a national movement control order instituted on March 18, aggressive testing and public health measures, 8,354 cases had been reported as of June $30,2020,{ }^{2}$ or $0.03 \%$ of the population. As of June 6 , most restrictions had been lifted as part of a phased recovery. We aimed to determine SARS-CoV-2 seroprevalence in residual serum samples collected at a teaching hospital serving Kuala Lumpur and Selangor state, which together have reported 4,483 cases (51.9\% of national cases), or $0.05 \%$ of the combined population. ${ }^{2}$

We retrieved 816 serum samples sent for diagnostic testing for non-respiratory infections (mainly dengue) and archived at $-20^{\circ} \mathrm{C}$. These were divided into periods according to dates of collection: pre-pandemic (JuneAugust 2019, $\mathrm{n}=228$ ), main wave (January 29 to April 14, 2020, $\mathrm{n}=327$ ) and post-wave (April 15 to June $6,2020, \mathrm{n}=261)$. For each period, between $17-65$ samples were included from every 10 -year age group $(<10$, $10-19,20-29,30-39,40-49,50-59,60-69$, and $>70$ years). The samples were from 368 females and 448 males.

Samples were first screened with an in-house indirect ELISA detecting IgG to SARS-CoV-2 receptor binding domain (RBD), and shown to be $100 \%$ sensitive for samples collected from 14 days post-onset of illness. ${ }^{3}$ Screen-seropositive samples were confirmed with a highly sensitive and specific (99.3-100\%) surrogate viral neutralization test (sVNT; cPass, GenScript, USA) based on total antibody-mediated blockage of ACE2 receptor-RBD interaction, ${ }^{4,5}$ which has received provisional authorisation from the Singapore Health Sciences Authority. A two-step testing process of screening with a highly sensitive assay and confirmation with a highly specific assay is useful for low-prevalence settings where seropositives have a low predictive value. ${ }^{5}$ 
These two assays were evaluated in our laboratory with the 228 (ELISA) or 26 (sVNT) pre-pandemic serum samples as negative controls and 35 samples collected from PCR-confirmed COVID-19 patients at least 16 days post-onset of illness. Sensitivity and specificity rates for the screening ELISA were $97.1 \%$ and 88.6\%, respectively. For the confirmatory sVNT assay, sensitivity and specificity rates were $100 \%$, after increasing the inhibition cut-off from $20 \%$ to $25 \%$, as suggested by the manufacturer after assessing background reactivity in our setting. Our two-step testing process thus utilised assays with 97\% sensitivity (screening) and 100\% specificity (confirmatory), exceeding the United States FDA-recommended minimum sensitivity of $90 \%$ and specificity of $95 \%$ for serology tests with emergency use authorisation. ${ }^{6}$ Crude seroprevalence rates are reported with $95 \%$ exact binomial confidence intervals (CI) approximated with Poisson distribution.

A total of $46(7.8 \%)$ main wave and post-wave samples screened positive, of which 3 were confirmed by sVNT. Two were from the main wave (seroprevalence 0.6\%; 95\% CI, 0.07-2.2\%) and 1 from the post-wave period $(0.4 \%, 95 \%$ CI, 0.01-2.1\%) (Figure 1). Two were from males aged in their 20 s with previous diagnoses of COVID-19. The third was from a 65-year-old man with a 7-day history consistent with COVID-19, who was not tested for SARS-CoV-2. As rates for the main wave and post-wave periods were similar, they were combined to give a crude seroprevalence rate of $0.5 \%$ (95\% CI, 0.1-1.5\%). Using 2019 age- and genderstratified population data for Kuala Lumpur and Selangor from the Department of Statistics, Malaysia (http://pqi.stats.gov.my/searchBI.php), a direct age-standardised seroprevalence rate was calculated as $0.4 \%$ (95\% CI, $0-0.93 \%)$.

This study is potentially limited by bias arising from use of residual inpatients serum. However, residual serum can provide similar estimates of seroprevalence to cohort studies ${ }^{7}$ and is a convenient option when preliminary data is needed during a lockdown. The rate may also be underestimated because antibodies may take 2 weeks to appear and may be undetectable in some mild or asymptomatic cases.

The age-standardised seroprevalence of $0.4 \%$ for Kuala Lumpur and Selangor found in this study is higher than the period prevalence of confirmed cases of $0.05 \%$. This is consistent with other seroprevalence studies revealing 6-24 times more COVID-19 infections than are reported. ${ }^{8}$ As this was a single centre study, a more extensive national serosurvey is necessary to confirm our preliminary indication that Malaysia has experienced limited SARS-CoV-2 transmission to date. With little herd immunity, Malaysia remains highly susceptible to COVID-19 as we emerge from lockdown. Continued vigilance in surveillance and public health measures are critical pending availability of an effective vaccine.

\section{Conflicts of interest}

CWT is a patent holder in cPass. The other authors declare no competing interests.

\section{Ethics statement}

This study was approved by the University Malaya Medical Centre medical ethics committee (no. 2017116$5794)$.

\section{Acknowledgments}

We are grateful to Professor Lin-Fa Wang, Duke-NUS Medical School, Singapore, and GenScript Biotech for providing the sVNT testing kits. 


\section{REFERENCES}

1. Kwok KO, Lai F, Wei WI, et al. Herd immunity - estimating the level required to halt the COVID-19 epidemics in affected countries. $J$ Infect. 2020;80(6):e32-e33. https://doi.org/10.1016/j.jinf.2020.03.027

2. Ministry of Health Malaysia. 2020. Press statement from the Director-General of Health, 30 June 2020: updates on the coronavirus disease 2019 (COVID-19) situation in Malaysia. https://kpkesihatan.com/2020/06/30/kenyataan-akhbar-kpk-30-jun-2020-situasi-semasa-jangkitanpenyakit-coronavirus-2019-covid-19-di-malaysia/. Accessed on August 8, 2020.

3. Chia WN, Tan CW, Foo R, et al. Serological differentiation between COVID-19 and SARS infections. Emerg Microbes Infect. 2020;9(1):1497-1505. https://doi.org/10.1080/22221751.2020.1780951

4. Tan CW, Chia WN, Chen MIC, et al. A SARS-CoV-2 surrogate virus neutralization test (sVNT) based on antibody-mediated blockage of ACE2-spike (RBD) protein-protein interaction. Nat Biotechnol. 2020. https://doi.org/10.1038/s41587-020-0631-z.

5. Bond K, Nicholson S, Lim S, et al. Evaluation of serological tests for SARS-CoV-2: Implications for serology testing in a low-prevalence setting. J Infect Dis. 2020; https://doi.org/10.1093/infdis/jiaa467.

6. U.S. Food \& Drug Administration. In Vitro Diagnostics EUAs - Serology Template for Commercial Manufacturers (updated June 26, 2020). https://www.fda.gov/media/137698/download. Accessed on August 8,2020 .

7. Kelly H, Peck HA, Laurie KL, et al. The age-specific cumulative incidence of infection with pandemic influenza H1N1 2009 was similar in various countries prior to vaccination. PLoS ONE. 2011;6(8):e21828. https://doi.org/10.1371/journal.pone.0021828

8. Havers FP, Reed C, Lim TW, et al. Seroprevalence of antibodies to SARS-CoV-2 in 10 sites in the United States, March 23-May 12, 2020. JAMA Intern Med. 2020. https://doi.org/10.1001/jamainternmed.2020.4130.

\section{Figure legend}

FIGURE 1. Epidemic curve of COVID-19 in Malaysia in 2020 from the first reported case on January 25 to June 30. The periods of serum sampling are shown (main wave, January 29 to April 14; post-wave, April 15 to June 6$)$ with crude seroprevalence rates (95\% CI). 


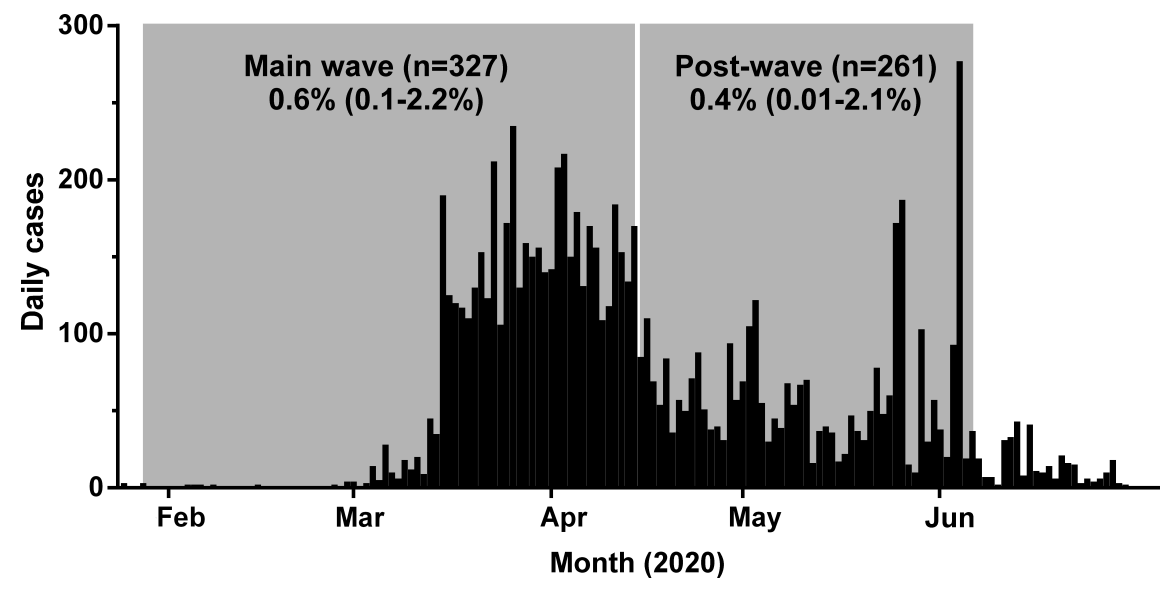

